\title{
Outcome of pregnancies in women with prosthetic mechanical heart valves.
}

\author{
Poonam Sharma (Koirala)
}

\author{
Department of Obstetrics \& Gynaecology, Institute of Medicine, Tribhuvan University Teaching Hospital. Kathmandu,Nepal \\ Corresponding author, Poonam Sharma (Koirala) \\ Department of Obstetrics \& Gynaecology \\ Institute of Medicine, Tribhuvan University Teaching Hospital. Kathmandu,Nepal, E-mail: punishita@gmail.com
}

\begin{abstract}
Background and Aims: Rheumatic heart disease is still prevalent in Nepal. Many young women had to undergo cardiac surgery before or at their childbearing age. Due to teratogenic effect and maternal risk, pregnancy is generally not advised to those women who are under oral anticoagulant for prosthetic mechanical heart valves. We aim to evaluate the pregnancy outcome and thromboembolic events during pregnancy in patients with mechanical heart valves.

Methods:This was a prospective, descriptive study conducted at the department of Obstetrics and Gynaecology of Tribhuvan University Teaching Hospital from January 2012 to June 2015. All the pregnant patients with mechanical heart valves who attended our antenatal clinic were included in this study.

Results: : Eighteen women attended our antenatal clinic at different gestational age. Among them fifteen women arrived early and took LMWH in first trimester as well after 36 weeks or before delivery. Three women attended antenatal clinic after the first trimester and took LMWH only in third trimester before delivery. In those patients who took LMWH at first trimester, all had favorable outcome except one. In those women who continued oral anticoagulants in first trimester one had intrauterine fetal death. Conclusion: : Our study suggests that pregnancies in prosthetic mechanical heart valves women have a favorable outcome even in the low-resource country like Nepal.
\end{abstract}

Key words: Low Molecular Weight Heparin; Mechanical Heart Valve; Pregnancy; Warfarin.

\section{Introduction:}

Rheumaticheart disease (RHD) is still a common cause of valvular malfunction in Nepal.1 It often warrants mechanical heart valve replacement and most of the female undergo valve replacement before or at the childbearing age. Patients with prosthetic mechanical heart valves require long life anticoagulation, which can be achieved using warfarin, unfractionated heparin,low molecular weight heparin (LMWH) or combination of above. In Nepal many valve replacement surgeries are done but only few women take the risk of becoming pregnant. Pregnancy in women with mechanical heart valves is associated with an increased risk of maternal and fetal complications and anticoagulation management of these women is difficult. Thromboembolism rates in pregnant women with prosthetic valves have been reported to be between $7 \%-23 \%$ per patient per year and half of these thromboembolism episodes arise from valve thrombosis.2The risk of valve thrombosis due to inadequate anticoagulation has to be balanced against the risk of direct harm to the fetus by the anticoagulant used along with the risk of hemorrhage to both mother and fetus.

We aim to evaluate the thromboembolic and hemorrhagic complications during pregnancy, maternal and fetal outcome in women with prosthetic mechanical heart valves in Department of Obstetrics \& Gynaecology, Institute of Medicine, Tribhuvan University Teaching Hospital(TUTH),
Kathmandu,Nepal.

\begin{abstract}
Methods
It was a prospective, single centre, observational study conducted from January 2012 to June 2015 in the obstetrics and gynaecology department of TUTH. The study was approval from the hospital ethical review board. Patients with valve replacement were counseled as soon as they reported conception. They were properly informed regarding use of anticoagulants and their complications. Complications due to inadequate dosing were also fully explained. The risks of warfarin and LMWH were explained and all patients who agreed for use of LMWH and warfarin were enrolled in the study after acquiring written consent for all those patients. Exclusion criteria for this study were pregnant women who wished to terminate pregnancy in first trimester of pregnancy, had miscarriage before 20 th week of gestation, with bioprosthetic valves and with valve repairs. As per the recommendations from an American consensus conference on antithrombotic therapy for patients with mechanical heart valves we applied third regime: Unfranctionated Heparin (UFH) or LMWH therapy until the 13 th week of gestation, followed by warfarin until the middle of the third trimester and then restarting UFH or LMWH therapy until delivery. 3
\end{abstract}


For those patients, who attended antenatal clinic at or before 6th week of pregnancy, LMWH (enoxaparin) in therapeutic doses was given from 6 weeks of pregnancy till 13 th week. After 13 th week warfarin was given until 36 th week. Dose of warfarin was adjusted at regular intervals based on individual's Prothrombin time and internationalized ratio (PT/INR) reports. Deliveries were planned at 36th week of gestation and LMWH was restarted after stopping warfarin. For elective deliveries, LMWH was stopped twelve hours before deliveries. In cases of spontaneous onset of labour or induction of labour before 36th week, warfarin was immediately stopped and fresh frozen plasma was administered. For those patients who attended antenatal clinic only at second trimester, anticoagulation management was similar to those who attended at first trimester as well. In absence of hemorrhagic complication, $\mathrm{LMWH}$ was reinstituted six hours after vaginal delivery and twelve hours after caesarian section. Warfarin was restarted in overlapping dose with LMWH on the third day of delivery and switched to warfarin alone after obtaining INR in therapeutic range, i.e.2.0-3.5, according to the valve surgery.

Adverse fetal outcome was defined as intrauterine fetal death, intracranial bleeding and still birth. Adverse maternal outcome was defined as cardiac failure, antepartum haemorrhage, postpartum haemorrhage and maternal death. Thromboembolic events were defined as valve thrombosis, need of valve replacement during pregnancy, stroke during or postpartum period. Those patients were followed during and after delivery and above mentioned complications recorded.

Data were computed and analyzed using SPSS ver.15 as a statistical tool. Numerical data were expressed in mean+/SD.

\section{Results:}

During the study period, 10437 deliveries were conducted at the TUTH. Among them 18 women fulfilled the inclusion criteria and were enrolled in this study. The mean age of those women was $24 \pm 3.5$ years. Details of demographic data, types of valvular surgeries and birth weight of live child were given in Table 1 .

Table 1. Demographic data and outcome.

$\begin{array}{llll}\text { SN } & \text { Descriptions } & \text { Results +/-SD(range) } \\ \text { 1. } & \text { Mean age (years) } & \text { 24+/-3.53 (18-33) } \\ \text { 2. } & \text { Gestational age (weeks) } & 36.17+/-4.02 \\ \text { 3. } & \text { Mean parity } & 1.17+/-0.39(1-2) \\ & \text { MVR } & 8(44.4 \%) \\ \text { 4. } & \begin{array}{l}\text { Types of Valve } \\ \text { replaced }\end{array} & \text { AVR } & 3(16.7 \%) \\ & \text { DVR } & 7(38.9 \%) \\ \text { 5. } & \begin{array}{l}\text { Birth weight of live child } \\ \text { (Kg) }\end{array} & 2.78+/-0.45(1.75-3.75)\end{array}$

AVR: Aortic valve replacement, MVR: Mitral valve replacement, DVR: Double valve replacement.

Fifteen women attended antenatal clinic early in the first trimester and received LMWH from the sixth week to thirteenth week whereas three women appeared late and as they were already in second trimester and wished to continue pregnancy so warfarin was continued after counseling about the risk of fetal anomalies.

Two patients were in atrial fibrillation, all had normally functioning prosthetic heart valves and all had normal left ventricular ejection fraction. Only one patient had mild pulmonary hypertension which did not warrant any medication.
Three pregnancies had intrauterine growth retardation (IUGR). There were two cases of intrauterine fetal death (IUFD), in one woman who took warfarin throughout the first trimester and another woman who followed the prescribed regimen. Two women went into spontaneous labour and one had to undergo elective caesarian section for IUGR before 36th week. Two women had maternal complications, namely postpartum hemorrhage.

There were two cases of fetal complication i.e. intrauterine fetal death. The modes of deliveries are illustrated in Chart 1. Among those women who underwent lower segment caesarian section (LSCS), two were due to failed induction, two had IUGR, two had fetal distress where as one had breech presentation.

\section{Modes of deliveries}

\section{$\square$ NVD $\square$ Vacuum Assisted VD $\square$ LSCS}

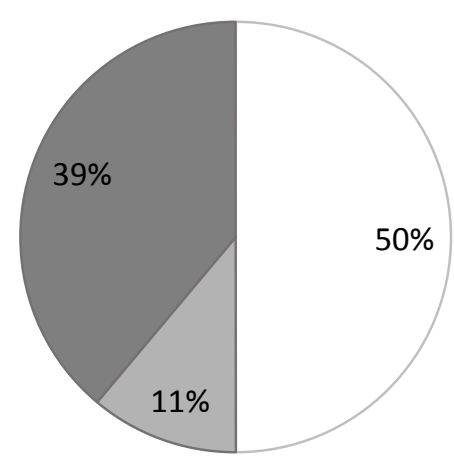

Chart 1: Bar Diagram of Mode of deliveries.NVD: Normal Vaginal Delivery, VD: Vaginal Delivery, LSCS: Lower Segment Caesarian Section.

Out of three patients who took warfarin in first and second trimester, one had IUFD. In that woman INR was found to be more than therapeutic range (INR= 7.3). Fourteen women who took LMWH at first trimester and again at third trimester had good fetal outcome. One woman had IUFD at 27 th week and delivered vaginally.

Sixteen women had normal postpartum period. Two women developed preeclampsia late in third trimester and had atonic postpartum hemorrhage which was managed conservatively. None of the patient had valve thrombosis during and after delivery. There was no maternal death, thromboembolic event or need of valve replacement during pregnancy or at postpartum period. Patients were followed up within a week of discharge. They continued their regular INR check-up and dose readjustment of warfarin at cardiac clinics.

\section{Discussion:}

In Nepalese population the incidence of RHD is common and many valve replacement surgeries are being done in young girls before or at childbearing age. 1 Either due to improper counseling by cardiac surgeons or lack of understanding of seriousness of pregnancy while taking oral anticoagulant patient reports in our department with pregnancy, often late.

Though the use of LMWH, particularly enoxaparin has proven efficacy in anticoagulation of patients with prosthetic valves, there have been reports of both clinical success 4 and failure5 with it in pregnant woman. Among three types of anticoagulant management recommended from an American 
consensus conference on antithrombotic therapy for patients with mechanical heart valve who wished to be pregnant, we chose third regimen. 3 It was cost effective and compliance was good in our population. In this study proper counseling was done after conception and they were explained about the side effects of various anticoagulants. Mc Lintock et al.conducted a retrospective observational study of three anticoagulation regimes: enoxaparin before 6 weeks gestation, between 6 and 12 weeks gestation and oral anticoagulation throughout pregnancy. 6 With enoxaparin use, thromboembolic complications were identified in seven (14.9\%) pregnancies, of which five were related to the poor compliance or sub-therapeutic anti-Xa levels. No such complications occurred in the 20 pregnancies, where enoxaparin use was therapeutic. There were also no thromboembolic complications with oral anticoagulation, although there were four warfarin-attributed fetal losses. Thus, the authors identified that therapeutic-dose enoxaparin is associated with a low risk of thrombosis and good foetal outcomes, but close monitoring is essential. A review of 81 pregnancies in 75 women with mechanical heart valves treated with LMWH during pregnancy reported a high $(8.6 \%)$ rate of valve thrombosis prompting one LMWH manufacture to issue a warning regarding the use of its product for this purpose. This followed the early termination of a study that found a higher than expected thrombosis rate in LMWH group compared with UFH group, although this was subsequently thought to be due to inadequate dosing and monitoring rather than the drug itself.7

Warfarin has not only teratogenic effects but it is also associated with spontaneous fetal intracranial bleeding and fetal death when it is taken in second and third trimester of pregnancy. Warfarin use in first trimester may result in fetal abnormalities such as fetal nasal hypoplasia, abnormalities of central nervous system, mental retardation and stippling of epiphyses of long bones. The overall incidence of warfarin-induced embryopathy is reported to be about $5 \%$, we did not encounter such in our study, whether in women taking warfarin throughout the second trimester or in women who were switched to LMWH.8 However this number is too small to suggest or to comment on warfarin embryopathy.

In general, vaginal delivery is a better option than caesarean section for women with heart disease. The risk of bleeding, infection, and clotting complication is less and vaginal delivery is not associated with acute shift in blood volume which occurs in cesarean section. However a long labour and difficult vaginal delivery is much more morbid than a cesarean section and in many cases it is preferable to avoid the hemodynamic effects of long labor and delivery by performing a quick cesarean section.

Postpartum hemorrhage complicates approximately $4 \%$ of vaginal and $6 \%$ of caesarean section. 9 In our study the incidence is high $11.1 \%$ in comparison to normal population probably as both the two patients were high risk patients, associated with both heart disease and hypertension. Preeclampsia complicates $5-8 \%$ of pregnancies and is a major cause of maternal morbidity. 10 Both coagulation and fibrinolytic activity is increased in normal pregnancy but coagulant activity is further enhanced in pre-eclamptic women.

There was study which had high incidence of valve thrombosis while being treated with LMWH.. However we did not have such problem. This may be due to use of warfarin in second and early third trimesters minimizing the use inferior anticoagulant like LMWH.

Yinon et al used LMWH throughout the pregnancy and found that there was increased risk of maternal cardiac events.11 Similarly a high incidence of maternal thromboembolism and death has been reported both by Sadler12 and Salazar.13 In contrary to those reports, we did not have any maternal cardiac event probably due to our regimen.

Single centre study with very small number of patients is the limitation of our study

\section{Conclusion:}

Our study reflects that favorable obstetric outcome cab be achieved in women with prosthetic mechanical heart valves provided early attendance in antenatal clinic and close monitoring at tertiary care centre.

\section{References:}

1. Regmi PR, Wyber R. Prevention of Rheumatic fever and heart disease: Nepalese experience. Global Heart 2013; 8 (3): $247-52$.

2. Roudaut R, Serri K, Lafitte S. Thrombosis of prosthetic heart valves; diagnosis and therapeutic considerations. Heart 2007; 93:137-42.

3. Bonow RO, Carabello BA, Chatterjee K et al. 2008 focused update incorporated into the ACC/AHA 2006 guidelines for the management of patients with valvular heart disease: a report of the American College of Cardiology/American Heart Association Task Force on Practice Guidelines. JACC 2008;52(13): 1-142.

4. Lec LH. Low molecular weight heparin for thromboprophylaxis during pregnancy in two patients with mitral valve replacement, Thromb Haemostas 1996; 76: 62830.

5. Rowan JA, McGowan LME, Raudkvivi PJ, et al. enoxaparin treatment in 12 mechanical heart valves during pregnancy. Am J Obstet Gynaecol 2001; 185; 633-7.

6. McLintock C, McCowan LM, North RA. Maternal complications and pregnancy outcome in women with mechanical prosthetic heart valves treated with enoxaparin. BJOG Int J Obstet Gynaecol.2009;116:1585-1592.

7. Brennard JE, Walker ID, Greer IA. Anti activated Factor $\mathrm{x}$ profiles in pregnant women receiving antenatal thrombophylaxis with enoxapar8in. Acta Haematol 1999; 101: 53-5.

8. Chan WS, Anand S, Ginsberg JS. Anticoagulation of pregnant women with mechanical heart valves: a systematic review of the literature. Arch Intern Med 2000;160: 191-6.

9. Dildy GA 3rd. Postpartum hemorrhage: new management options. Clin Obstet Gynecol 2002;45(2):330-44.

10. Cunningham FG, Gant NF, Lenovo KJ, et al. Hypertensive disorders in pregnancy. In Williams Obstretics, 21 st ed. New York. McGraw-Hill, 2001, 567-618.

11. Y Yinen, Samuel C, Chelsie Warshafsky, et. al. Fetal and maternal cardiac complications. Am J Obster Gynaecol 2009; 104(9):1259-63.
Cite this article as: Poonam Sharma. Outcome of pregnancies in women with prosthetic mechanical heart valves. Nepalese Heart Journal 2016; 13 (1):- 5-7. 